\title{
Von der Ethik-Kommission zur gewissenhaften Organisation
}

\author{
Entscheidungen in Unternehmen können nicht \\ nur an ökonomischen Kriterien, sondern auch an \\ moralischen Werten ausgerichtet werden. Dazu \\ werden zunehmend Kodizes und externe Kontrol- \\ len eingeführt. Doch diese Ansätze reichen nicht \\ aus. Denn dabei werden Organisationen als trivia- \\ le Systeme betrachtet, denen ein Gewissen als \\ interne Funktion nicht zugetraut wird. \\ Von Paul Reinbacher
}

U nternehmen entdecken zunehmend ihre umfassende, über ökonomischen Erfolg hinausgehende Verantwortung für ihr die Umwelt betreffendes Tun oder Unterlassen, die sogenannte Corporate Responsibility. Sie beginnen, sich organisationstypisch, das heißt in Form von Planung, Entscheidung und Kontrolle, mit Moral auseinanderzusetzen. Dazu formulieren sie Ethik-Kodizes, richten Stellen wie Ethik-Kommissionen ein, unterwerfen sich heteronomen Kontrollen externer Agenturen, betätigen sich als Wohltäter, indem sie Projekte finanzieren, die mit ihrem Geschäft mehr oder weniger in Zusammenhang stehen und so weiter.

Meist tun sie dies zwar keineswegs aus moralischer Überzeugung, sondern im Sinne eines Moral Marketing aus handfestem strategischen Interesse, also der Sicherung der eigenen Existenzgrundlagen zum Beispiel durch Beruhigung von Stakeholdern oder auch nur zwecks Imagebildung und Profilierung gegenüber ihren Kundinnen. Aber immerhin: Sie tun es.

Unabhängig davon, ob Aktivitäten zu Corporate Social Responsibility (CSR) von ethischen Überlegungen getragen sind oder instrumentellen Hintergedanken der ökonomischen Logik dienen, sind die meist trivialen, mechanistischen Vorstellungen die ihnen zugrunde liegen, problematisch oder zumindest kurzsichtig. Und das nicht nur hinsichtlich der bisweilen illusorischen Vorstellungen von den Möglichkeiten und Erfolgen einseitiger Bindung, die anderen theoretisch wie praktisch die Gewinne überlassen (Beckmann/Pies 2007).

Demgegenüber können in Unternehmen beziehungsweise Organisationen die Voraussetzungen für moralische Verantwortung in Gestalt der drei Freud'schen Funktionen Idealbild, Selbstbeobachtung, Gewissen identifiziert werden (Freud 1989). Damit können die Erwartungen hinsichtlich autonomer Moralität jenseits eines egoistischen Utilitarismus oder ökonomischer Strukturzwänge neu formuliert werden. Denn, wie Ortmann aufzeigt: „Warum ist der Gegensatz zum Nutzenstreben nicht, wie es die meisten Ökonomen suggerieren, Altruismus, sondern moralische Pflicht? Weil Altruismus ebenfalls eine Variante des Strebens nach Nutzen ist, nur eben: des Nutzens anderer, die dem Altruisten am Herzen liegt.“ (Ortmann 2004)

Bereits unweit ausgetretener Pfade stellt man fest, dass Unternehmen als soziale Systeme sehr wohl in der Lage sind, erstens, ein Idealbild von sich selbst zu entwerfen, zweitens, sich selbst zu beobachten, um Abweichungen zwischen Soll und Ist festzustellen sowie drittens, diese Abweichungen auf komplexe Weise, und das heißt eben gewissenhaft, zu bewältigen. Diese Fähigkeiten sind grundsätzliche, bestandsnotwendige Erfordernisse von Organisationen (Reinbacher 2009).

\section{Lösung und Teil des Problems}

Am Beginn von CSR-Projekten stehen üblicherweise Analysen der Organisationskultur, in deren Rahmen für das Unternehmen typische Werte und handlungsleitende Prinzipien identifiziert werden sollen. Ziel ist dabei, eine Antwort auf die Frage nach der individuellen Identität zu finden, wobei diese in den unhinterfragt angewandten Entscheidungsprämissen vermutet wird (Schein 1985, Luhmann 2000). Im Zuge dieses Reflexionsprozesses stellt sich jedoch meist früher oder später heraus, dass die gefundenen, dahinterliegenden Werte universell, allgemein und abstrakt sind. Zufall? Wohl kaum!

Werte lassen sich nicht nach Belieben wählen. Einerseits, weil mit ihnen implizit ein Anspruch der Allgemeingültigkeit erhoben wird. Andererseits, weil sie zumindest in einer aufgeklärten, säkularen Moral nicht dogmatisch durch Hierarchie oder historisch durch Tradition festgelegt, sondern nur durch Argumentation mit guten Gründen bestimmt werden können (Strasser 2004). Damit aber drängt sich das Problem auf, dass man sich nur entweder zur Moral als solcher mit ihrem gesamten Werteuniversum bekennen oder sich von ihr verabschieden kann. Kein Unternehmen, kein soziales System ist in der Lage, Werte im Unterschied zu anderen sozialen Normen, Bräuchen oder Konventionen zu definieren (Reinbacher 2009). Entweder etwas ist ein Wert, oder nicht. Abhängig ist dies allein von seiner Begründung vor einem universellen Auditorium. Ist diese gegeben, so steht der Wert nicht mehr zur Disposition!

Daraus erklärt sich letzten Endes die hohe Vergleichbarkeit von Leitbildern und Kodizes, sofern diese in einem offenen Entwicklungsprozess lange genug diskutiert und mit Blick auf ein universelles Auditorium, wie man es von der iterativen und falliblen Wahrheitssuche in der Wissenschaft kennt, argumentiert werden. So wenig wie wissenschaftliche Erkenntnis lässt sich $\rightarrow$ 


\section{„Überall wo formal kommuniziert und entschieden wird, ist die Gefahr der Täuschung allgegenwärtig."}

eben auch moralische Erkenntnis subjektiv beziehungsweise systemintern definieren. Unternehmen unterscheiden sich nicht durch abstrakte Werte, denen sie in Gestalt des moralischen Horizonts gegenüber stehen, sondern durch die Praxis, in die jene individuell implementiert werden. Also dadurch, wie sich das alltägliche Handeln und Entscheiden an diesen moralischen Werten orientiert, kommt die ideale Einzigartigkeit zum Ausdruck, wie bereits die frühen Kulturvergleiche von Hofstede gezeigt haben. Derselbe Wertekodex wird eben individuell von Unternehmen umgesetzt. Daher scheint es angebracht, anstelle von Organisationskultur, die sich in definierten Werten ausdrücken will, von Organisationskultivierung, die einen Prozess der individuellen Aneignung von Werten beschreibt, zu reden (Reinbacher 2008).

Schließlich und endlich sind Werte wie Gerechtigkeit oder Gleichbehandlung noch nicht gut geeignet, konkretes Handeln anzuleiten. Erforderlich ist deren Auslegung und Konkretisierung jeweils für eine bestimmte Situation (Ortmann 2003). Und auch diese Situation selbst muss interpretiert werden, da sich keine zwei Anwendungsfälle völlig gleichen.

\section{Unternehmenseigene Fähigkeiten}

Unternehmen bedürfen als Organisationen offensichtlich stets der Fähigkeit, mit allgemeinen normativen Vorgaben umzugehen beziehungsweise diese in der Praxis in individuelle Idealbilder zu transformieren. Ebenso selbstverständlich bilden sie Mechanismen der Selbstbeobachtung aus, zumal sie von ihrem Management ständig in strukturelle Spannung zwischen aktuell Gegebenem und pozentiell Möglichem versetzt werden (Baecker 2000, 2003). Also können sie nicht nur prinzipiell, sondern auch konkret in Fragen der Ökonomie wie der Moral Abweichungen des tatsächlichen Geschehens von normativen Ansprüchen feststellen. Wie wären sonst Sanktionen bei Verstößen gegen Vorgaben, oder wie wären Steuerungsmaßnahmen beziehungsweise Feedback-Schleifen im Zuge von Soll-Ist-Vergleichen möglich? Formal kommt diese Aufgabe dem Management $\mathrm{zu}$, doch bekanntlich wäre es naiv, die informelle Kommunikation zu unterschätzen. Gerade von der Hierarchie nicht sanktioniertes abweichendes Verhalten wird oft abseits des offiziel- len Dienstwegs in sozialen Räumen umso heftiger und emotionaler diskutiert. So ist ja auch die Debatte um organisationales Lernen unter anderem deshalb stark positiv verzerrt, weil sie zu wenig berücksichtigt, was in Organisationen informell sehr rasch gelernt wird, nämlich wie Vorgaben und Routinen zu umgehen sind, beziehungsweise aufgetretene und unter der Hand festgestellte Abweichungen am besten zu vertuschen oder kaschieren sind.

Allerdings ist mittlerweile hinlänglich bekannt, dass nicht jede Abweichung per se dysfunktional für das Gesamtsystem ist und deshalb steuernde Eingriffe auslösen muss (Luhmann 1964). Allgemeine Regeln wie Unternehmensvorschriften, Verhaltensnormen oder moralische Werte bedürfen einer selbst nicht regelbaren Konkretisierung und konstituieren sich daher vollends erst in ihrer Anwendungssituation. Daher müssen sie immer irgendwie anders aussehen. Regeln müssen also dehnbar bleiben und der abweichungsverhindernde, möglichst exakte Dienst nach Vorschrift bringt das System rascher zum Erliegen, als die ständigen und notwendigen kleinen Regelverletzungen, die es erst am Laufen halten, beziehungsweise derer die Organisation als brauchbare Illegalität so dringend bedarf.

\section{Gewissen als moralischer Gradmesser?}

Obwohl auch die Vorstellung vom Gewissen als einem moralischen Gradmesser weit verbreitet ist, lässt sich individuell im Alltag erleben und nun auch mit den obigen skizzenhaften Ausführungen erklären, weshalb die Dinge etwas verwickelter liegen. Es handelt sich nämlich wohl kaum um einen trivialen Feedback-Mechanismus, der Abweichungen von festgelegten moralischen Sollwerten im System feststellt und aussteuert. Schon von Controlling als dem betriebswirtschaftlichen Gewissen wird zusätzlich zur Rück- auch die Vorkoppelung im Sinne von Feed-forward, Vorsteuerung erwartet.

Demgegenüber ist es eine laufende Balance zwischen Regeln und den Situationen ihrer Anwendung, die sich wechselseitig bestimmen (Ortmann 2003; Reinbacher 2009): Es gibt im Anwendungsfall nicht nur Konflikte zwischen Werten, sondern auch unterschiedliche Möglichkeiten, einem Wert zu folgen. Und Situationen sind ihrerseits nicht einfach gegeben, sondern es gibt mehr als eine Möglichkeit ihrer Interpretation. Daher ist stets zu fragen, erstens was eine Regel bedeutet, zweitens was eine Situation bedeutet, drittens was jene Regel in dieser Situation für das aktuelle Handeln und Entscheiden bedeutet und viertens im Sinne des Feed-forward, welche Auswirkungen dieser konkrete Anwendungsfall im Gegenzug für die Zukunft der allgemeinen Regel hat.

Außerdem lässt sich die situationsabhängige Regelanwendung nicht ihrerseits durch Regeln definieren. Es muss also letztlich aus Sicht eines jeden Regelwerks offen bleiben, für welche Abweichungen vom Management und von der formalen Kommunikation Regelungen getroffen werden, und welche demgegenüber zu ignorieren sind. 
Werden solchermaßen problematische Konflikte zwischen moralischen Regeln oder deren Anwendung behandelt, das heißt explizit zum Thema der formalen Kommunikation gemacht, so stellt dies nichts anderes als ein funktionales Äquivalent auf der Ebene der Organisation zum Schuldbewusstsein des Individuums, also zur ausdrücklichen Beschäftigung mit konfligierenden Anforderungen dar.

Darüber hinaus taucht aber so einiges, was als Abweichung beziehungsweise Differenz und damit als Konflikt formell nicht behandelt wird, ins Informelle, stärker affektiv-emotional Strukturierte ab (Luhmann 1964). Hier lässt sich dann schon von einem Äquivalent zu Schuldgefühlen sprechen, die im Gegensatz zum Schuldbewusstsein eher unter der Oberfläche angesiedelt sind. Sobald die normativen Selbst-Ansprüche anerkannt sind, werden diese erfahrungsgemäß von den Mitarbeiterinnen und Mitarbeitern als Maßstab an das Handeln und Entscheiden eines Unternehmens angelegt. Dies ist auch unabhängig davon, ob sie in Leitbildern oder ähnlichen Dokumenten niedergeschrieben beziehungsweise vom Management berücksichtigt werden (Reinbacher 2009).

Auf diese Weise werden neben der anderweitig beobachtbaren organisationalen Praxis die informellen Gespräche am Kaffeeautomaten meist zu besseren Sonden für die Gewissenhaftigkeit eines Unternehmens, also für den Umgang mit Abweichungen von moralischen Werten, als das offizielle Reporting über Corporate Responsibility. Denn überall, wo formal kommuniziert, das heißt entschieden wird, ist dies mit der Gefahr von Täuschung verbunden (Cohen et al. 1972; Luhmann 2000). Und es zeigt sich, dass Unternehmen in der Lage sind, mehr oder weniger intendiert und reflektiert mit Abweichungen zu jonglieren. Dabei werden diese Abweichungen bei der Interpretation oder Neudefinition von Regeln berücksichtigt oder eben ignoriert, um damit schließlich gewissenhaft zu agieren.

\section{Was können Management und Beratung leisten?}

Nachdem Unternehmen als organisierte soziale Systeme offensichtlich einem gemeinsamen moralischen Horizont an Werten gegenüberstehen und daneben nicht nur zur Selbstbeobachtung, sondern auch zur Balance zwischen stets unvollständigen Regeln sowie deren situationsspezifischer Anwendung fähig sind, stellt sich vor allem die Frage, was Management als Systemfunktion in puncto Gewissenhaftigkeit der Organisation beziehungsweise unter dem Titel Gewissensmanagement im Unternehmen zu leisten imstande ist.

Wie man beim Menschen weder Wissen noch Gewissen implementieren, sondern nur im Zuge von Erziehung möglichst gute Rahmenbedingungen für dessen autonome, eigenständige Entwicklung schaffen kann, muss auch im Falle von Organisationen eine autonome Entwicklung an die Stelle formaler Struktur- und Kontrollmechanismen treten. Die Entwicklung kognitiver Fähigkeiten und die Ausbildung moralischer Urteils- kraft sind nichts anderes als Teile eines Kultivierungsprozesses, der die Berücksichtigung von Wissen und Werten in der täglichen Praxis sowie die Ausbildung der drei internen Funktionen Idealbild, Selbstbeobachtung und Gewissen beinhaltet (Reinbacher 2008; 2009).

\section{Gewissenbildung in Organisationen}

Sich zu Werten und damit zu Moral insgesamt zu bekennen mag der erste Schritt sein (Idealbild), deren Implementierung zu überwachen und zu steuern ein zweiter (Selbstbeobachtung), doch erst deren laufendes Ausbalancieren in interpretationsbedürftigen Situationen mit widersprüchlichen Anforderungen zeigt Gewissenhaftigkeit - bei jenen Menschen wie auch bei jenen Organisationen, die Komplexität, wie sie aus diesen Ambivalenzen entsteht, nicht apodiktisch verbannen, sondern produktiv zu nutzen wissen. Gefragt ist gerade aus diesem Grund eine Rahmensteuerung, die den Kontext für einen Umgang mit Komplexität fördert (Reinbacher 2009). Denn die Orientierung an Werten macht die Welt unübersichtlicher und nicht einfacher.

Dass Kontrolle, Strafen oder Anreize diese kulturelle Entwicklung unterstützen und beschleunigen können, soll damit keineswegs bestritten werden. Allerdings hat sich gezeigt, dass solch einer mechanistischen Vorstellung prinzipielle Grenzen und nicht nur empirisch-faktische Umsetzungshürden gesetzt sind. Weil moralische Urteilskraft eben anders funktioniert und die Anwendung von moralischen Regeln selbst nicht geregelt werden kann. Formale Strukturen und deren Kontrolle sind in Unternehmen unabdingbar, nicht zuletzt, da sie Organisationen erst zu solchen machen. Diese wollen in Entscheidungszusammenhängen und ganz besonders bei der Berücksichtigung moralischer Werte als Prämissen als Teil eines Gesamtkonzepts verstanden werden (siehe Abbildung 1).

Die Rahmensteuerung zur Gewissenbildung in Organisationen zeichnet sich durch die folgenden Aspekte aus.

I Caution als umsichtige Bedachtnahme auf informale Kommunikation und auf das, was sich unterhalb des offiziell- $\rightarrow$

Abbildung 1: Aspekte der Rahmensteuerung zur Gewissensbildung im System Organisation/Unternehmen

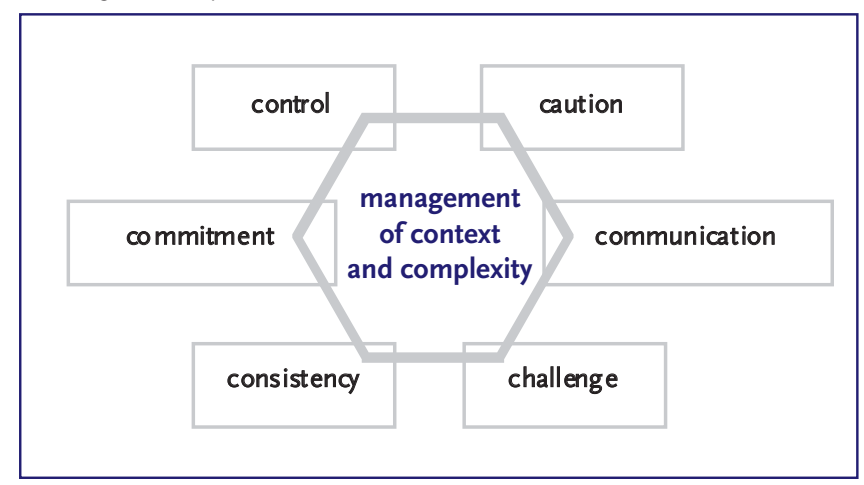

Quelle: eigene Darstellung 


\section{„Die Anwendung von moralischen Regeln in Unternehmen kann nicht durch Mechanismen wie Kontrollen oder Strafen durchgesetzt werden"}

organisatorischen Kontrollradars von Soll-Ist-Vergleichen und Reports abspielt. Schließlich wird nicht alles illegale Verhalten aufgedeckt und formaler Behandlung unterzogen. Deshalb lohnt es sich, ein Ohr auch für schwache Signale zu haben.

- Consistency als Vermeidung struktureller Überforderung, wie sie durch Appelle an moralisches Handeln bei gleichzeitiger Gratifikation ökonomischer Egoismen oder unmoralisch erzielter Ergebnisse entsteht.

- Commitment, das sich in Engagement, Vorbildwirkung und Konsistenz der Worte und Taten insbesondere der Führungskräfte ausdrückt. Als moralischer Hygienefaktor wirkt es dissonanten Wahrnehmungen entgegen und fördert Glaubwürdigkeit von Struktur und Kultur. Schließlich können Führungspersonen Themen und Beiträgen unterschiedliches Gewicht verleihen.

- Challenge als Bereitschaft und Mut zum Hinterfragen des Üblichen, also beispielsweise von scheinbar naturgesetzlichen utilitaristischen Logiken und ökonomischen Systemzwängen. Damit verbunden ist ein Akzeptieren der in Abweichungen und in positiven Abweichungsverstärkungen enthaltenen Risiken.

- Communication ganz allgemein als probates Mittel zur Erhöhung des Reflexivitätsgrades eines Systems. Extensive Beteiligung der Mitglieder einer Organisation verhindert Versuche einer Delegation von Gewissenhaftigkeit an einzelne Bereiche und Funktionen.

Im Übrigen vermutete schon Freud, dass die Selbstkritik des Gewissens mit der Selbstbeobachtung, auf die sie gebaut ist, zusammenfallen könnte. Und nachdem die Beratung in Organisationen die Unterstützung ihrer Selbstreflexion zum Ziel hat, ist auch von ihr ein Beitrag zur Gestaltung moraladäquater organisationaler Kontexte, Strukturen und Kulturen zu erwarten (Reinbacher 2008).

Wo Organisationen darauf drängen, Entscheidungsprozesse dogmatisch durch unentschiedene Prämissen beziehungsweise Werte abzubrechen, tendiert Beratung zu einer Entschleunigung, indem sie die Kontingenz dieser scheinbaren Sicherheit aufdeckt, und damit zeigt, dass hier sehr wohl eine Entscheidung möglich wäre, die auch anders ausgehen könnte.

Gemeinsam schaffen Manager und Berater gewissenhafter Unternehmen die Voraussetzungen, dass diese Organisationen nicht vorschnell die ökonomische Logik mit ihrem WerteUniversum als gegeben hinnehmen und sich in ihren Entscheidungen dieser unterwerfen - auch wenn sich damit schnell Eindeutigkeit herstellen ließe und es daher dem System die liebste Unsicherheitsreduktion wäre. Vielmehr ist es ihnen möglich, Varietät nicht nur zu erhalten, sondern vorübergehend sogar zu erhöhen. Damit leistet Gewissenhaftigkeit zur Viabilität in unübersichtlichen Umwelten einen Beitrag, indem sie die Organisation mehr Optionen erkennen lässt.

\section{Literatur}

Baecker, D.: Ausgangspunkte einer soziologischen Managementlehre. In: Soziale Systeme 6, 1/2000. S. 137-168.

Baecker, D.: Management im System. In: Baecker, D.: Organisation und Management. Frankfurt am Main 2003. S. 256-292.

Beckmann, M. / Pies, I.: Freiheit durch Bindung. Zur ökonomischen Logik von Verhaltenskodizes. In: Schmalenbachs Zeitschrift für betriebswirtschaftliche Forschung 59, 8/2007. S. 615-645.

Freud, S.: Die Zerlegung der psychischen Persönlichkeit. In: Freud, S.: Vorlesungen zur Einführung in die Psychoanalyse. Frankfurt am Main 1989. S. 496-516.

Luhmann, N.: Funktionen und Folgen formaler Organisation. Berlin 1964. Luhmann, N.: Organisation und Entscheidung. Opladen 2000.

Cohen, M. / March, J. / Olsen, J.: A Garbage Can Model of Organizational Choice. In: Administrative Science Quarterly 17, 1/1972. S. 1-25.

Ortmann, G.: Regel und Ausnahme. Paradoxien sozialer Ordnung. Frankfurt am Main 2003.

Ortmann, G.: Als Ob. Fiktionen und Organisationen. Wiesbaden 2004

Reinbacher, P.: Werte, Kultur und die »Kultivierung « der Organisation. Zugleich ein Beitrag zur CSR-Debatte. In: Zeitschrift Führung und Organisation 77, 6/2008. S. 360-366.

Reinbacher, P.: Gewissensmanagement in Organisationen. Möglichkeiten im Umgang mit Corporate Social Responsibility. Wiesbaden 2009.

Schein, E. H.: Are You Corporate Cultured? In: Personnel Journal 65, 11/1985. S. 83-96.

Strasser, P.: Gut in allen möglichen Welten. Der ethische Horizont. Paderborn 2004

\section{AUTOR + KONTAKT}

Dr. Paul Reinbacher ist wissenschaftlicher Mitarbeiter am FH Campus Wien im Bereich Sozialwirtschaft und soziale Arbeit sowie Lehrbeauftragter an den Universitäten Innsbruck und Linz.

Paul Reinbacher, FH Campus Wien, Departement Soziales, Favoritenstr. 226, 1100 Wien, Österreich. E-Mail: Paul.Reinbacher@fh-campuswien.ac.at 
(c) 20I0 Authors; licensee IÖW and oekom verlag. This is an article distributed under the terms of the Creative Commons Attribution Non-Commercial No Derivates License (http://creativecommons.org/licenses/by-nc-nd/3.o/), which permits unrestricted use, distribution, and reproduction in any medium, provided the original work is properly cited. 